\title{
Study of the propagation of ultra-high energy cosmic rays in extragalactic magnetic fields
}

\author{
Lima E. $\mathbf{F}^{\star a}$, M. A. Leigui de Oliveira $^{b}$ and C. J. Todero Peixoto ${ }^{b}$ \\ ${ }^{a}$ Departamento de Astronomia, UFRGS, Porto Alegre, RS, Brazil \\ ${ }^{b}$ Universidade Federal do ABC, Santo André, SP, Brazil \\ E-mail: Eliade.limadufras.br teiquidufabc.edu.br, \\ toderocjeufabc.edu.br
}

\begin{abstract}
We studied the propagation of high energy charged particles (protons with energy between $10^{18}$ and $10^{21} \mathrm{eV}$ ) in the extragalactic magnetic field with a simple model, without energy loss, taking into account only the diffusion of the particles under the influence of the extragalactic magnetic field.
\end{abstract}

4th School on Cosmic Rays and Astrophysics,

August 25-September 04, 2010

Sao Paulo - Brazil

${ }^{*}$ Speaker. 


\section{Introduction}

Recently, a correlation between the arrival directions of events with energy greater than 57 $\mathrm{EeV}$ and the positions of catalogued Active Galactic Nuclei has been observed by the Pierre Auger Observatory [四]. However, the determination of the sources for individual cosmic rays is very difficult, because they suffer deflections during their travel to Earth due to interactions with the galactic and extragalactic magnetic fields.

The knowledge of the magnetic fields in extragalactic space is quite limited. The observational technique is the measurement of the Faraday rotation, which also requires knowledge of the electron density between the source and the observer. Very high fields, of order of and exceeding $1 \mu G$, have been observed in clusters of galaxies on $M p c$ scales. The average random extragalactic magnetic field does not exceed $1 \mathrm{nG}$ if the coherence length $l_{c}$ of the field is $1 \mathrm{Mpc}$, in the order of magnitude of the average distance between the galaxies [ [ $]$ ].

Large-scale magnetic fields are generally assumed to have a cellular structure. Namely, the magnetic field $\vec{B}$ is supposed to be constant over a domain of size $L_{c}$, randomly changing its direction from one domain to another but keeping approximately the same strength [B]].

\section{Random Magnetic Field}

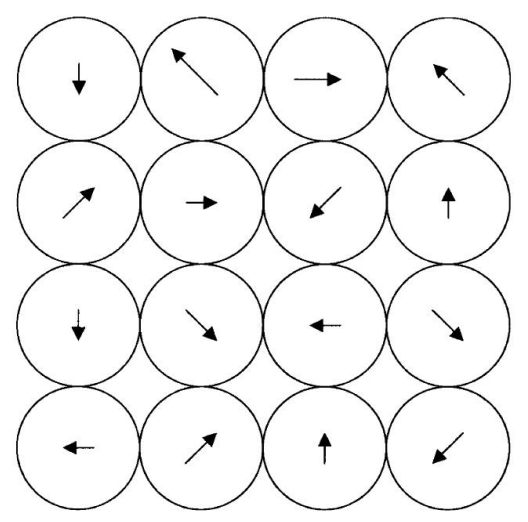

Figure 1: A sketch of a cellular magnetic field structure, valid for cells in a supercluster of galaxies, or cells inside a cluster of galaxies.

Models employing a random magnetic field $\mathrm{B}$ in cells predicted an increase in the rotation measure variance with redshift. Searches for such a $B_{\text {ran }}$ component yielded an upper limit of $1 \mathrm{nG}$ out to a redshift of $z \sim 3.5$, using spherical cell sizes of $\sim 1 M p c$ (figure $\mathbb{W}$ ). A similar study for a random $\mathrm{B}$, using thin elongated web-like filaments of size $\sim 1 M p c$ in voids, has led to a $B_{\text {ran }}<1 \mu G$ [四]. Blasi et al. [G] assuming that the cosmological inhomogeneities are described by the observed Lyman-alpha forest distribution and that the magnetic field direction is random from cell to cell and its strength in a cell is $B \sim n^{2 / 3}$. For a cell size $l_{c} \sim 50 M p c$, they found the primordial $B<6 n G$, or even smaller if there have been subsequent amplification by battery or dynamo processes. Sethi [目] employed a two-point correlation function to study moments as a function of the redshift, for distances up to $z \leq 3$, and found no signal or oscillations due to the presence of a random magnetic 
field on scales $>10 \mathrm{Mpc}$, corresponding to fields $<3 \times 10^{-8} \mathrm{G}$. He noticed that no signal or anisotropy occasioned by random magnetic fields on the $3 K$ microwave background radiation were found, implying fields $<3 n G$ at this large redshift (last scattering surface).

\section{Propagation of UHECRs using Frenet-Serret formulas}

If an observer moves with a particle with charge $q$, rest mass $m_{0}$ and Lorentz factor $\gamma$ in a magnetic field $\vec{B}$, the Frenet-Serret equations can be used to describe the equations of motion of this particle, with the advantage of this observer to be in a non-inertial frame, i.e., the Frenet-Serret frame rotates with the particle and there is no need to change the reference frame to perform the calculations. From the equation for the Lorentz force, we have:

$$
\frac{d}{d t} \vec{v}=\frac{q}{m_{0} \gamma}\left(\vec{v}_{\|} \times \vec{B}\right)+\frac{q}{m_{0} \gamma}\left(\vec{v}_{\perp} \times \vec{B}\right),
$$

where $v_{\|}$and $v_{\perp}$ are, respectively, the velocity components parallel and perpendicular to the field. The velocity parallel to the magnetic field is given by:

$$
\vec{v}_{\|}=\frac{(\vec{v} \cdot \vec{B})}{B^{2}} \vec{B}
$$

Substituing the equation (B.2) into the equation (B.]) we can write:

$$
\frac{d}{d t} \vec{v}=\vec{v}_{\perp} \times \vec{\omega}_{B}
$$

where $\vec{\omega}_{B}$ is known as the angular gyrofrequency and is given by: $\vec{\omega}_{B}=\frac{q \vec{B}}{m_{0} \gamma}$.

The equation (B.3) implies that the particle accelaration is perpendicular to $\vec{v}_{\perp}$ which in turn points in the direction of motion. Hence, we can define the Frenet-Serret frame as:

$$
\begin{gathered}
\vec{T}=\frac{\vec{v}_{\perp}}{\left|\vec{v}_{\perp}\right|}, \\
\vec{N}=\frac{\vec{T}^{\prime}}{\left|\vec{T}^{\prime}\right|}=\frac{\frac{d \vec{v}_{\perp}}{d t}}{\mid \frac{d \vec{v}_{\perp} \mid}{d t}=\frac{\left(\vec{v}_{\perp} \times \vec{\omega}_{B}\right)}{\left|\left(\vec{v}_{\perp} \times \vec{\omega}_{B}\right)\right|},} \\
\vec{M}=\vec{T} \times \vec{N}=\frac{\vec{v}_{\perp}}{\left|\vec{v}_{\perp}\right|} \times \frac{\left(\vec{v}_{\perp} \times \vec{\omega}_{B}\right)}{\left|\left(\vec{v}_{\perp} \times \vec{\omega}_{B}\right)\right|}=-\frac{\vec{\omega}_{B}}{\omega_{B}} .
\end{gathered}
$$

where $\vec{T}$ is the unit vector tangent to the particle trajectory, $\vec{N}$ is the vector normal to the particle trajectory and $\vec{M}$ is the cross product of $\vec{T}$ and $\vec{N}$.

The Larmor radius $R_{L}$ of the particle is inversely proportional to curvature $\kappa$, where $R_{L}=\frac{\omega_{B}}{v_{\perp}}$, consequently:

$$
\kappa=\frac{q B}{m_{0} \gamma v_{\perp}}
$$

The torsion $\tau$ is defined by:

$$
\tau=-\vec{N} \cdot \frac{d \vec{M}}{d t}
$$


Assuming that the magnetic field $\vec{B}$ is constant, $\frac{d \vec{M}}{d t}=0$ and $\tau=0$, which describes a particle in a circular trajectory ${ }^{1}$ on a plane, named osculating plane. Taking the time derivative of the other two vectors, one can easily show that:

$$
\frac{d \vec{T}}{d t}=v_{\perp} \kappa \vec{N} \quad \text { and } \quad \frac{d \vec{N}}{d t}=-v_{\perp} \kappa \vec{T}
$$

which we can summarize in the following matrix equation:

$$
\frac{d}{d t}\left[\begin{array}{l}
\vec{T} \\
\vec{N} \\
\vec{M}
\end{array}\right]=v_{\perp}\left[\begin{array}{ccc}
0 & \kappa & 0 \\
-\kappa & 0 & \tau \\
0 & \tau & 0
\end{array}\right]\left[\begin{array}{l}
\vec{T} \\
\vec{N} \\
\vec{M}
\end{array}\right]=v_{\perp}\left[\begin{array}{ccc}
0 & \kappa & 0 \\
-\kappa & 0 & 0 \\
0 & 0 & 0
\end{array}\right]\left[\begin{array}{l}
\vec{T} \\
\vec{N} \\
\vec{M}
\end{array}\right]
$$

To propagate the particle according to the equations B.9, we first define a time bin $\Delta t$ much smaller than the period of a revolution $\left(\Delta t \ll \frac{2 \pi R_{L}}{v_{\perp}}\right)$ and then calculate the next position of the particle by:

$$
\vec{r}=\vec{r}_{0}+\vec{v} \Delta t
$$
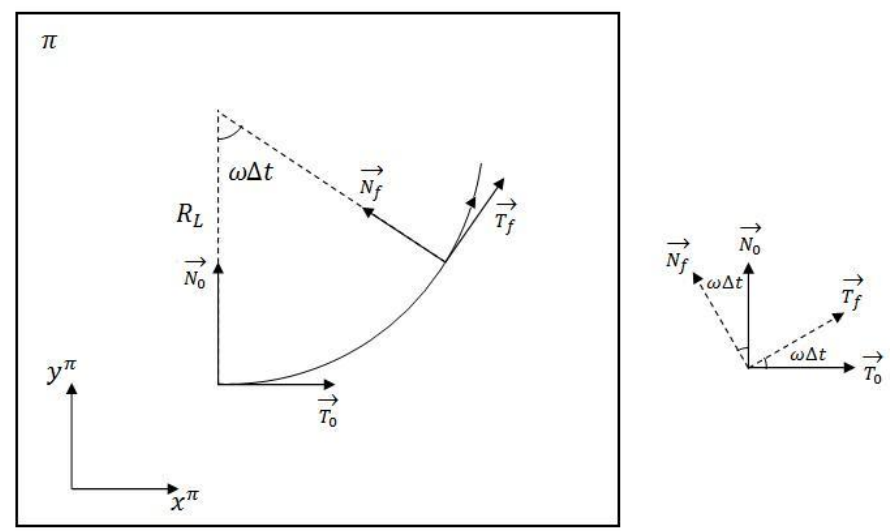

Figure 2: Osculating plane $\pi$, generated by $\vec{T}$ and $\vec{N}\left(\pi: \vec{R}_{0}+\mu \vec{T}+v \vec{N}\right)$, showing the particle movement after one time bin.

Now, defining the osculating plane $\pi$, as shown in figure ఐ, we will have for each step:

$$
\begin{cases}x_{f}^{\pi}=x_{0}^{\pi}+R_{L} \sin (\omega \Delta t) & , \text { in the direction of } \vec{T}_{0} \\ y_{f}^{\pi}=y_{0}^{\pi}+R_{L}[1-\cos (\omega \Delta t)] & , \text { in the direction of } \vec{N}_{0} .\end{cases}
$$

And after one iteration:

$$
\left\{\begin{array}{l}
\vec{T}_{f}=\cos (\omega \Delta t) \vec{T}_{0}+\sin (\omega \Delta t) \vec{N}_{0} \\
\vec{N}_{f}=-\sin (\omega \Delta t) \vec{T}_{0}+\cos (\omega \Delta t) \vec{N}_{0}
\end{array}\right.
$$

such that the new velocity will be:

$$
\vec{v}_{f}=\vec{v}_{\|}+v_{\perp} \vec{T}_{f}
$$

\footnotetext{
${ }^{1} \mathrm{~A}$ circle of radius $r$ has zero torsion and curvature $1 / r$.
} 


\section{Our model}

We simulated protons propagating inside a cubic Universe of $(200 M p c)^{3}$, where the unitary cell is a cube of side $l_{c}=1 \mathrm{Mpc}$. For this proposal, we assumed in each cell random orientations for the field, but constant intensities of $\sqrt{3} n G$, as observed in the figure B.

The proton is launched from the center of the Universe, with fixed energies between $10^{19}$ and $10^{21} \mathrm{eV}$, such that its initial speed is given by:

$$
v=c \sqrt{1-\gamma^{-2}} \quad, \text { where } \quad \gamma=\frac{E}{m_{0} c^{2}} .
$$

The motion is determined by the equations $3 ., 1], 3.5$ and 3.54 .

Then, we compared these propagations with those in an anisotropic and uniform Universe (figure 耳), with $B_{x}=B_{y}=B_{z}=1 \mathrm{nG.}$

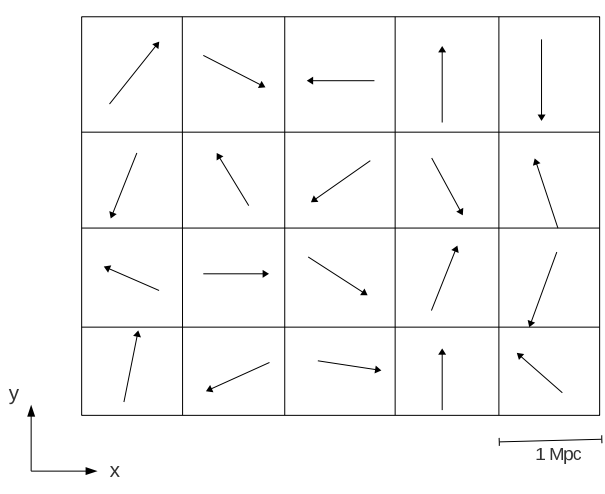

Figure 3: Random magnetic field

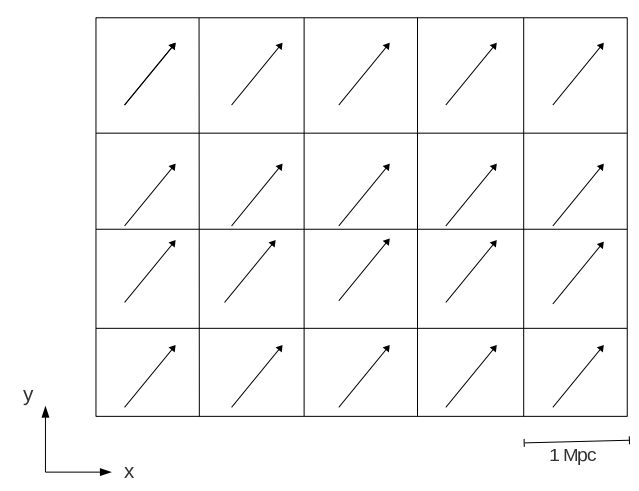

Figure 4: Uniform magnetic field

The step size between iterations, $\Delta s$, has been taken to be a very small fraction of the instantaneous Larmor radius of the particle: $\Delta s=10^{-6} R_{L}$. Since, for ultra-relativistic particles, the parameter $\beta=v / c$ taken from equation 4 . d demands several decimal places to be calculated, we implemented in the code the GNU MPFR library [ $[8]$ to achieve the needed accuracy.

\section{Results}

The resulting simulated trajectories for some selected energies are given in figures $\square$, $\square$ and $\square$. One can see the expected resulting trajectory for the uniform field (plots at the right boxes), i.e., particles propagating in a spiral, according to their energies. However, for the random orientation fields (plots at the left boxes), the particles tend to be reflected in the regions of the borders, where the discontinuities of the fields take place.

For each energy value, we have propagated 100 protons. (We intend to increase the statistics to 1000 particles/energy.) 

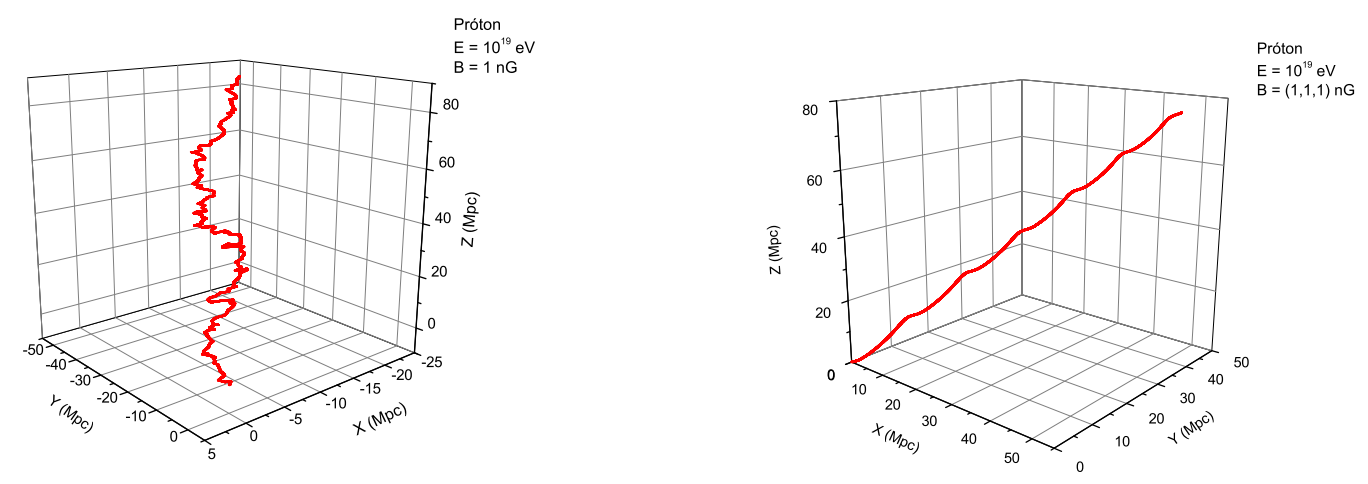

Figure 5: Proton propagation with energy $E=10^{19} \mathrm{eV}$ : (left) propagation in the random magnetic field $B=\sqrt{3} n G$ and (right) propagation in the uniform magnetic field $B_{x}=B_{y}=B_{z}=1 n G$.
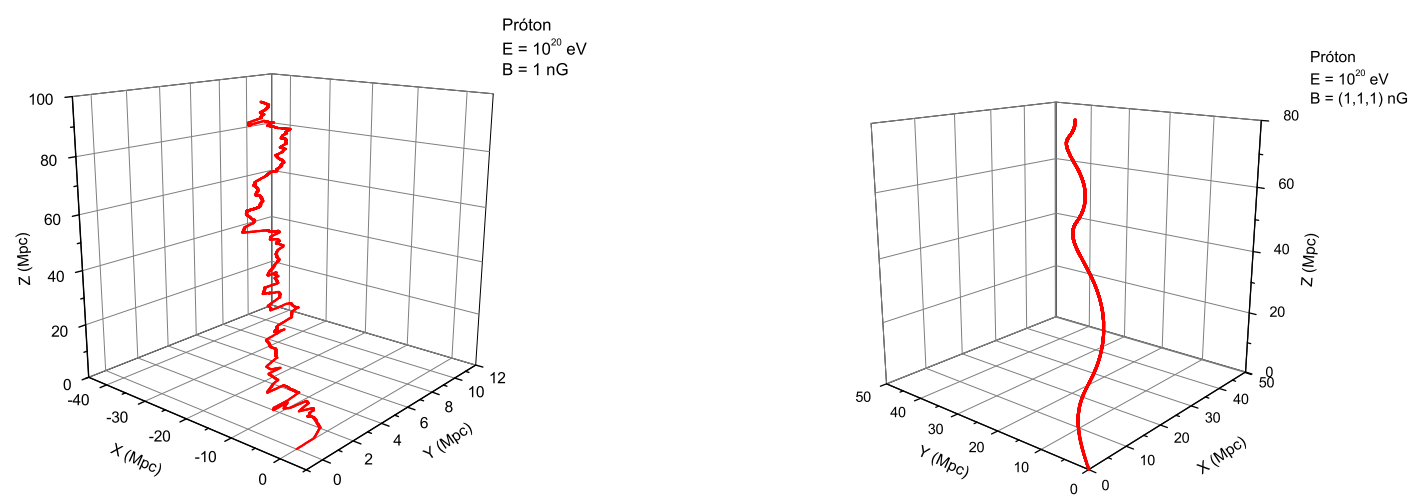

Figure 6: Proton propagation with energy $E=10^{20} \mathrm{eV}$ : (left) propagation in the random magnetic field $B=\sqrt{3} n G$ and (right) propagation in the uniform magnetic field $B_{x}=B_{y}=B_{z}=1 n G$.
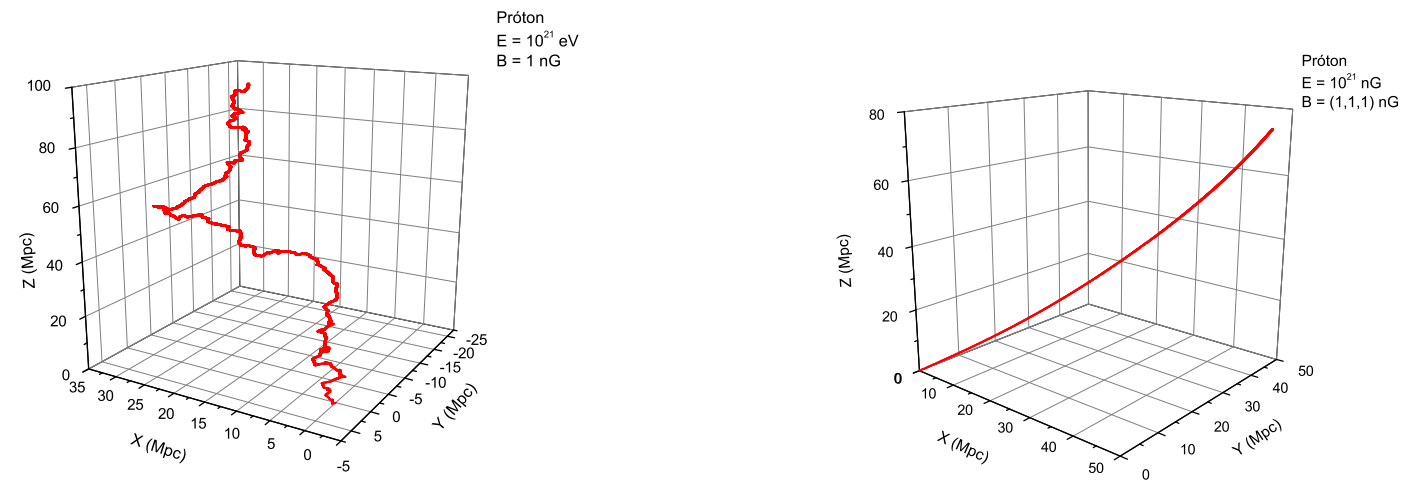

Figure 7: Proton propagation with energy $E=10^{21} \mathrm{eV}$ : (left) propagation in the random magnetic field $B=\sqrt{3} n G$ and (right) propagation in the uniform magnetic field $B_{x}=B_{y}=B_{z}=1 n G$. 


\section{Conclusions}

We propagated the particles through the Lorentz force equation in the formalism of the FrenetSerret equations [ []].

We did not expect the sharp deviations in the trajectory of the protons in the case of the random field. In order to verify these results, we ran the same protons in uniform fields and the results were consistent with those present in the scientific literature, i.e., the higher the energy, the smaller the particle deviation that will suffer during the simulated trajectory [9]. The work is progress in order to smooth the field discontinuities in the regions of the cells borders.

\section{Acknowledgments}

We thank UFABC for financial and computacional support and G. Medina-Tanco for useful discussions and suggestions.

\section{References}

[1] The Pierre Auger Collaboration, Science 318, 938 (2007).

[2] Todor Stanev, Astrophys. J 479, 290 (1997).

[3] A. De Angelis, M. Persic e M. Roncadelli, Mod. Phys. Lett. A23, 315 (2008).

[4] D. Ryu, H. Kang e P. L. Biermann, A \& A 335, 19 (1998).

[5] P. Blasi, S. Burles e A. Olinto, Astrophys. J. 514, L79 (1999).

[6] S. K. Sethi, MNRAS 342 962, (2003).

[7] E. Kreyszig, Differential Geometry, Dover Publications Inc. (1991).

[8] http://www.MPFR.org/.

[9] G. Sigl, F. Miniati e T. A. Ensslin, Phys. Rev. D 68, 043002 (2003). 